\title{
The Potential for Combination Treatment Using STAT-C Drugs
}

\author{
David L. Wyles, MD
}

Corresponding author

David L. Wyles, MD

Division of Infectious Diseases, University of California, San Diego, 9500 Gilman Drive, MC 0711, La Jolla, CA 92093, USA.

E-mail:dwyles@ucsd.edu

Current Hepatitis Reports 2009, 8:167-174

Current Medicine Group LLC ISSN 1540-3416

Copyright @ 2009 by Current Medicine Group LLC

Given the limitations of pegylated interferon (IFN) and ribavirin (RBV) therapy for chronic hepatitis $\mathrm{C}$ virus (HCV) infection, new antiviral medication and treatment approaches are sought. Specifically targeted antiviral therapies for hepatitis C (STAT-C) refers to the use of these new inhibitors, either in combination with IFN and RBV or other STAT-C agents, to improve therapy for HCV. Although many classes of inhibitors are being developed, NS3 protease and NS5B polymerase inhibitors are likely to be among the first STAT-C agents approved. The preclinical and clinical characteristics of HCV protease and polymerase inhibitors are reviewed in this article. Strengths and weaknesses of each class in the context of developing future all-STAT-C regimens are explored, with a particular focus on viral resistance and the prospects for eliminating IFN and/or RBV.

\section{Introduction}

Therapy for chronic hepatitis $\mathrm{C}$ virus (HCV) infection saw incremental improvements over the past decade with the introduction of pegylated interferons (PEG-IFN), weight-based ribavirin (RBV), virologic stopping criteria, and improved management of side effects. Despite these advances, the rate of sustained virologic response (SVR) for genotype $1 \mathrm{HCV}$ following PEG-IFN/RBV therapy is about $45 \%$ [1]. Gains from further dose and duration modification of IFN-based therapy are likely minimal and fail to address the large number of patients for whom IFNbased therapy is contraindicated [2]. Additionally, several difficult-to-treat populations (eg, African Americans and HIV-1-coinfected individuals) respond significantly less well to IFN-based therapy [3,4].

The shortcomings of IFN-based therapy and a significant drug discovery effort focusing on HCV resulted in an array of virus-specific inhibitors at various stages of development. Specifically targeted antiviral therapy for hepatitis C (STAT-C) refers to the use of compounds targeting HCV proteins or, in some cases, required cellular cofactors to treat persons with hepatitis C. Although initial approaches focus on the addition of STAT-C agents to PEG-IFN/RBV, the ultimate goal is a potent, welltolerated regimen of multiple STAT-C drugs that can be administered to an expanded population, including those with contraindications to IFN-based therapy. Before this goal can be realized, several hurdles must be overcome, including the vast genetic diversity of $\mathrm{HCV}$, rapid development of drug resistance, and drug interactions in populations (eg, HIV-1-coinfected persons receiving antiretrovirals). In this article, the feasibility and potential of STAT-C combination therapy are examined; major classes of inhibitors are addressed for their potential in future STAT-C combination therapy; and approaches to testing and implementing these regimens are discussed.

\section{Hepatitis C Virology: Implications for}

\section{Use of STAT-C Agents}

$\mathrm{HCV}$ is a positive-sense RNA virus of about 9600 nucleotides encoding a single polyprotein, which is then cleaved by host and virally encoded proteases to form functional viral proteins. HCV replication occurs in the cytoplasm within replication complexes that contain viral and cellular proteins. The viral NS5B RNA-dependent RNA polymerase has no proofreading mechanism, resulting in a swarm of closely related viral genomes (referred to as a quasispecies) within an infected person. As a result of this error-prone replication, HCV shows remarkable genetic diversity on a population level, with about $35 \%$ difference in nucleotide sequence between viral genotypes and up to $25 \%$ difference in subtypes [5]. This genetic diversity presents a challenge to the development and implementation of STAT-C agents.

Although the error-prone polymerase is a large factor in the development of resistance to STAT-C agents, other characteristics of the HCV life cycle further compound these issues. The amount of virus produced in an infected individual is estimated to be $10^{11}$ to $10^{12}$ virions per day; about 10 - to 100 -fold higher than that seen in HIV-1 [6•]. The more viral turnover that occurs, the higher the likelihood that preexisting resistance mutations will 


\section{Table 1. Key concepts for hepatitis C NS3 protease inhibitors}

Profound, rapid inhibition of HCV replication

$>3 \log$ by day 3 of therapy for most Pls

Adequate drug exposure is key to maximizing activity and limiting resistance

Exposure prioritized over dosing convenience at this stage of development

Rapid selection and outgrowth of resistant variants occurs during monotherapy

Significant inter- and intragenotype variability exists in the activity and resistance barrier of current clinical lead Pls

Cross-resistance and persistence of resistant mutants may limit sequential treatment options

Mutations R155K and A156T/V confer broad PI cross-resistance

Select resistant mutants can remain significant components of the viral quasispecies for years after exposure

HCV—hepatitis C virus; Pl—protease inhibitor.

quickly grow out under selective pressure. The HCV genome encodes a single polyprotein and does not have overlapping reading frames, relaxing the constraints on tolerated mutations compared with other chronic viral infections (eg, hepatitis B virus [HBV] and to a lesser extent HIV-1), which have overlapping reading frames. Finally, the life span of infected hepatocytes is shorter for HCV than HBV, with an estimated half-life of days to several months for HCV and months to years for HBV [6•]. In contrast, productively HIV-1-infected CD4 T cells have a half-life of about 1 day with rapid turnover, creating a large "replication space" for HIV when compared with HCV and HBV. One important difference between HCV and both HBV and HIV-1 is the lack of an integrated (HIV-1 proviral DNA) or latent (HBV cccDNA) cellular form that is capable of archiving resistance mutations and reestablishing active infection after antiviral therapy. This characteristic of HCV replication may increase the chances of successful STAT-C combination therapy; however, the impact of potential sanctuary sites (eg, the central nervous system) and prolonged persistence of relatively fit resistant mutants remains to be determined. In sum, these HCV viral characteristics suggest that resistance mutations will preexist and be selected rapidly during STAT-C antiviral therapy.

\section{Protease Inhibitors \\ Overview}

The HCV NS3 protease is essential for viral replication; by cleaving the single viral polyprotein at several sites, the protease creates functional viral proteins. Early recognition of this role in viral replication and the success of similar approaches in HIV-1 therapy led to an intense drug-discovery effort targeted at this protein [7]. However, structural characteristics of the protein proved challenging when specific and potent NS3 protease inhibitors (PI) were sought. The protease active site is shallow, relatively featureless, and exposed-characteristics that make the rational design of inhibitors difficult. Early successes focused on modeling candidate inhibitors after the authentic viral target and inhibitory cleavage products [8].
Following these design paradigms, two structural classes of NS3 PIs emerged: macrocyclic compounds and peptidomimetic ketoamides. The first proof-of-concept study in humans was conducted with BILN-2061 (a macrocyclic), and demonstrated the rapid and profound impact of PIs on HCV viral load [9]. Subsequent human trials confirmed these findings for macrocyclic and ketoamide PIs and provided additional insights. The major concepts regarding PI-based inhibition of HCV replication are discussed below and summarized in Table 1 .

\section{Illustrative compounds}

Several HCV PIs representing different chemical classes are at various stages in clinical trials. This review focuses on the compounds furthest along in clinical development and on those at earlier stages that appear to have unique and potentially advantageous characteristics pertaining to use in future STAT-C regimens. Two ketoamide HCV PIs-telaprevir and boceprevir-have commenced phase 3 trials after encouraging results in phase 2 trials in combination with PEG-IFN/RBV. The recently published Protease Inhibition for Viral Evaluation (PROVE)-1 and PROVE-2 studies (ClinicalTrials.gov numbers NCT00372385 and NCT00336479, respectively) demonstrated significant increases in SVR rates with 12 weeks of telaprevir, 750 $\mathrm{mg}$ three times daily, in combination with 24 weeks of PEG-IFN/RBV, compared with controls treated with PEG-IFN/RBV for 48 weeks (61\% vs $41 \%, P=0.02$ and $69 \%$ vs $46 \%, P=0.004$ respectively) $[10 \bullet \bullet, 11]$. Discontinuations because of adverse events, particularly rash, were higher in subjects treated with telaprevir compared with controls in the PROVE-1 (21\% vs $11 \%)$ and PROVE2 studies $(12 \%$ vs $7 \%)$. The Serine Protease Inhibitor Therapy-1 (SPRINT-1) study (ClinicalTrials.gov number NCT00705432) assessed boceprevir, $800 \mathrm{mg}$ three times daily, in combination with PEG-IFN/RBV for 28 or 48 weeks (boceprevir was administered for the entire time); SVR rates were $54 \%$ and $67 \%$ for the durations of 28 and 48 weeks $(38 \%$ control, $P=0.013$ and $P<0.0001$, respectively) and $75 \%$ with a 4 -week lead-in phase with PEG-IFN/RBV followed by 44 weeks of PEG-IFN/RBV/ boceprevir [12]. Treatment discontinuations because of 
adverse events were higher in the boceprevir arms $(11 \%$ in the 24 -week arm and $19 \%$ in the 48 -week arm) compared with controls $(8 \%)$.

Differences in trial design and patient populations studied preclude direct comparison of SVR rates between the trials; however, it appears that telaprevir and boceprevir comparably and significantly increase SVR rates in naïve HCV genotype 1-infected persons, albeit with increased rates of adverse events. Differences between the compounds focus on adverse effects and potential drug interactions because of different metabolic pathways. Tolerability in clinical practice may be an issue for both compounds, especially when coadministered with PEG-IFN/RBV.

The macrocyclic PI BILN-2061 validated the NS3 protease as an antiviral target, and several macrocyclic PIs are currently in clinical trials [13-15]. In early monotherapy and IFN combination studies, these inhibitors were potent and well tolerated, with the potential for once-daily dosing [15]. Finally, compared with other macrocyclic inhibitors, EA-063, a recently described PI, has smaller increases in median effective concentration $\left(\mathrm{EC}_{50}\right)$ with several common PI mutations (R155K and D168V) in vitro. The significance of this finding in vivo-particularly in patients who failed other HCV PI therapy-is unknown, given that these mutations still result in a 10 - to 500 -fold increase in $\mathrm{EC}_{50}$ in vitro to EA-063 [16]. These characteristics may offer a higher barrier to resistance rather than activity against established mutants.

\section{Resistance to protease inhibitors}

Resistance to HCV NS3 PIs is rapidly selected both in vitro and in patients during monotherapy-or, importantly, in combination with IFN when RBV is omitted or is used at low doses $[10 \bullet \bullet, 12,17,18,19 \bullet]$. Substitution of the alanine at position 156 with serine (A156S) and the aspartic acid at position 168 with valine (D168V) initially were described in vitro to confer resistance to ketoamide and macrocyclic inhibitors, respectively [17]. It was further shown that substitutions of valine or threonine at position 156 (A156T/V) conferred cross-resistance to ketoamide and macrocyclic inhibitors [18]. In vivo, the major HCV PI resistance mutations identified during telaprevir therapy were at serine 156 $(\mathrm{A} 156 \mathrm{~S}>\mathrm{A} 156 \mathrm{~T} / \mathrm{V})$, arginine $155(\mathrm{R} 155 \mathrm{~K} / \mathrm{T})$, threonine 54 (T54A), and valine 36 (V36M/A) [19•]. The R155K mutation has emerged frequently in published trials of PI therapy. In vitro, this mutation confers low-level resistance $(\sim 10 \times)$ to ketoamide inhibitors and high-level resistance to macrocyclic inhibitors $(>100 \times)$ while maintaining replicative fitness [20]. Based on in vitro and in vivo studies, the R155K mutation confers cross-resistance to all PIs currently in clinical trials and is fit enough that it occasionally was found as a majority species in chronically infected persons before PI exposure [21,22•]. In the PROVE-1 and 2 studies, the R155K mutation (either alone or in combination with $\mathrm{V} 36 \mathrm{M}$ ) was the most frequent mutation seen in genotype 1a patients with viral breakthrough or relapse after therapy; in genotype $1 \mathrm{~b}$, mutations at position 156 tend to be more common because changes at two nucleotides are required for the R155K mutation $[10 \bullet \bullet, 11]$. Mutations at position 36 confer low-level resistance in isolation; however, the significance of this mutation is that, when combined with mutations at either the 155 or 156 position, high-level resistance is observed with improved replication fitness [19•]. Long-term follow-up data are still being collected, but the R155K, T54A, and V36M mutations persisted for many months to years after the cessation of PI therapy in humans $[19 \bullet, 23]$.

Pretreatment with PEG-IFN/RBV for several weeks appears to decrease the rate of breakthrough and resistance development to PIs in early trials [12]. With lower replication levels, the ability of the virus to generate mutants is decreased and the outgrowth of preexisting mutants is hampered. PEG-IFN/RBV lead-in therapy may preemptively "pick off" preexisting resistant mutants that are less fit for replication in vivo. Poor or null responders to IFN-based therapy would not be expected to benefit from this approach. This approach is possible with PEG-IFN/RBV because of a lack of treatment-specific resistance mutations. In the future, trials composed solely of direct-acting STAT-C agents will require simultaneous combination therapy with inhibitors of several targets, and/or unique resistance mutations, to present a high combined-resistance barrier, as is the norm for tuberculosis or HIV-1 therapy.

\section{Potential role of protease inhibitors in STAT-C regimens} Given that PIs are farthest along in development, combined with their potency in vivo, they likely will be a prominent component of future STAT-C regimens, particularly for patients infected with genotype-1 HCV. Additionally, high levels (>20\% of the viral quasispecies) of preexisting resistant mutants appear to be relatively rare $(1 \%-5 \%$ of individuals with genotype 1) [21,22•]. Viral resistance may be an issue for patients failing an initial PI-based therapy because of cross-resistance conferred by mutations at positions 155 and 156 and prolonged survival of resistant mutants after exposure.

\section{Polymerase Inhibitors \\ Overview}

The HCV NS5B RNA-dependent RNA polymerase is essential for HCV replication, generating copies of both the minus and plus strands of viral RNA during replication. Crystal structures of the polymerase revealed a classic, right-hand configuration RNA polymerase with a highly conserved active site and nucleotide triphosphate binding tunnel [24]. Additional features of the polymerase with implications for STAT-C therapy include the lack of a proofreading mechanism (resulting in a high error rate), conformation change in the transition from initiation to a processive form, requirement of cellular cofactors for efficient replication (eg, cyclophilins), and the existence of multiple allosteric inhibitor binding sites distinct from the 


\begin{tabular}{|c|c|}
\hline Nucleoside inhibitors & Nonnucleoside inhibitors \\
\hline Potent activity with recent nucleosides & Modest activity, multiple targets \\
\hline 2-3 log RNA reduction with monotherapy & 1-2 log RNA reduction with monotherapy \\
\hline Broad cross-genotype activity & Limited cross-genotype activity \\
\hline Highly conserved polymerase active site & Variability in binding sites \\
\hline High barrier to resistance & Low barrier to resistance \\
\hline Mutants with low replicative fitness in vitro & Mutants relatively fit in vitro \\
\hline Resistance not yet demonstrated in vivo & Rapid resistance in vivo \\
\hline Preexisting resistance not noted & Preexisting resistance prevalent \\
\hline Potential interaction with ribavirin if coadministered & Inhibitors from many chemical classes \\
\hline
\end{tabular}

polymerase active site $[6 \bullet, 25,26]$. Because of the existence of the polymerase active site and multiple allosteric sites, two major classes of $\mathrm{HCV}$ inhibitors are directed at the NS5B polymerase: nucleoside polymerase inhibitors (NIs) and nonnucleoside polymerase inhibitors (NNIs). General characteristics of polymerase inhibitors as STAT-C agents are detailed below and summarized in Table 2 .

\section{Nucleoside polymerase inhibitors}

NIs target the NS5B polymerase active site by competitively inhibiting the incorporation of endogenous nucleotide triphosphates in the elongating RNA chain. They function as chain terminators once incorporated into the RNA molecule; however, because most compounds in this class possess a $3^{\prime} \mathrm{OH}$, they are termed nonobligate chain terminators [27]. NIs are attractive HCV inhibitors for several reasons, including the highly conserved nature of the polymerase active site, the proven utility of this class of inhibitors in other viral disease, and the high barrier to resistance described in vitro and in early clinical trials [24,28].

\section{Nonnucleoside polymerase inhibitors}

NNIs of the HCV polymerase bind to sites unique from the polymerase active site and inhibit its function in a noncompetitive manner with nucleotide triphosphates. At least four NNI binding sites with multiple chemical classes of compounds have been described to inhibit the purified polymerase and HCV replication in vitro [26]. Although specific mechanisms of action are not defined for all NNIs, many appear to exert their effect by inhibiting the conformational change necessary for the polymerase to go from initiation to elongation $[29,30]$.

\section{Illustrative compounds}

The first $\mathrm{HCV}$ nucleoside inhibitors described in vitro and tested in humans were the 2 '-methyl nucleosides, with the prototypical compound being a prodrug of $2^{\prime} \mathrm{C}$ methylcytidine [27,31]. Although initial clinical studies clearly demonstrated inhibition of HCV replication, early compounds were hampered by lack of potency and poor tolerability [31]. Second-generation NIs (eg, the 4'azido compounds) had improved potency in vivo (2-3 log decreases in HCV RNA during monotherapy), but were still plagued by poor tolerability, which limited exposure and increased relapse rates [32]. Recently, 2'-deoxy HCV nucleosides were described, and a related compound $\mathrm{R} 7128$ (a prodrug of 2'-deoxy-2'-fluoro-2'-C-methylcytidine) initiated phase $2 \mathrm{~b}$ trials in combination with PEG-IFN/RBV [33]. During a phase 1b study of R7128, $1500 \mathrm{mg}$ orally, twice daily, in combination with PEG-IFN/RBV for 4 weeks, $85 \%$ of subjects had an undetectable HCV viral load by the end of administration [34].

Several HCV NNIs were examined in human trials with limited success because of lack of efficacy and tolerability. Agents currently in clinical trials include ANA-598 (a palm site inhibitor) and VCH-916 (a thiophene 2-carboxylic acid derivative thumb site inhibitor) $[35,36]$.

\section{Resistance to polymerase inhibitors}

Resistance development to NI and NNI polymerase inhibitors is quite different, with NI resistance difficult to select in vitro and not yet seen in clinical trials. In contrast, NNI resistance is readily selected for both in vitro and in vivo. In vitro, the S282T mutation confers modest resistance (twoto threefold) to $2^{\prime}$-methyl substitutes nucleosides while significantly hampering the replicative fitness of replicons (5\%-10\% of wild-type) [28]. The S96T \pm N142T mutations confer resistance to the NI 4'-azidocytidine in vitro, again with modest changes in $\mathrm{EC}_{50}$ and a dramatic loss in replicative fitness (5\%) [28]. These features imply why these sites are highly conserved across all HCV genotypes and why mutants do not preexist in $\mathrm{HCV}$-infected patients to any significant degree [37•]. Nucleoside polymerase inhibitors (eg, the recently described 2'-deoxy-4'-azido nucleoside analogs) are active in vitro against the S282T and N96T mutants, with resistance mutations specific to this compound not reported [33].

Resistance to NNIs at all binding sites was described, and the allosteric sites can be identified by their signature resistance mutations [26]. Resistance to NNIs was reviewed [38]. Important features for their utility in STAT$\mathrm{C}$ regimens include a low genetic barrier to resistance, the ability of certain mutations to confer cross-resistance to other allosteric sites (eg, $\mathrm{C} 316 \mathrm{Y}$ confers resistance 
to inhibitors of both palm domains), and preexisting resistant mutants within the viral quasispecies in a high percentage of naïve patients [37•]. Furthermore, resistant variants retain much of their replication fitness in many instances, and dual resistance to a combination of a palm and thumb inhibitor can be readily selected in vitro [39].

\section{Potential role of polymerase inhibitors in STAT-C regimens}

NIs are particularly attractive as a component of an all-STAT-C regimen because of their high resistance barrier, broad genotype activity, and the low fitness/lack of preexistence of polymerase active site mutants. Although early trials validated the target, a lack of potency and side effects precluded the use of these early agents in STAT-C therapy. Current NIs are more potent; however, tolerability over 12 to 24 weeks of administration is unproven. One complicating factor for NIs, should RBV remain an essential component of HCV therapy, is the potential for intracellular phosphorylation interactions between RBV and nucleoside analogs [40].

The large number of allosteric binding sites and the diversity of chemical classes from which NNI inhibitors can be found increase the likelihood that viable anti-HCV drugs may emerge. However, issues such as limited and highly variable activity across and within genotypes may significantly limit the number of patients for which any given NNI may be useful. Pretherapy resistance testing (sequencing) may be necessary to successfully implement NNI therapy in a given patient, a scenario that is likely unnecessary for NIs or PIs.

\section{STAT-C Agents Directed at Other Viral Targets}

Inhibitors of several other HCV proteins including NS4A, the NS3 helicase, the HCV internal ribosome entry site, and NS5A are in various stages of development. Although they are not discussed here, the existence of multiple targets with multiple inhibitors increases the likelihood of realizing potent all-STAT-C regimens in the future. Targeting cellular proteins essential for $\mathrm{HCV}$ replication offers another means of inhibiting HCV replication. Current leading examples of such inhibitors include nitazoxanide and cyclophilin inhibitors. Nitazoxanide acts through increasing phosphorylation of double-stranded RNA-dependent protein kinase (PKR), ultimately inhibiting viral translation; nitazoxanide inhibits HCV replication in vitro and appears to augment the IFN response when coadministered [41]. Similar to IFN, no resistance mutations were found in HCV replicons during prolonged exposure. Preliminary results were reported in genotype 1 patients treated with combination therapy including nitazoxanide [42]. Nitazoxanide's clinical safety record (administered as an antiprotozoal for prolonged periods in humans) combined with a lack of described resistance make it a potential addition to STAT-C combination therapy, especially if it has an immune-modulating effect in vivo [41].
Cyclophilins are abundant host cellular proteins that interact with the HCV RNA polymerase and are necessary for efficient HCV replication [25]. Debio 025 is a nonimmunosuppressive cyclophilin inhibitor that Is a potent HCV inhibitor in vitro and in vivo $(-4.61 \log$ for Debio $025,600 \mathrm{mg}$, combined with PEG-IFN vs $-2.49 \log$ for PEG-IFN alone for 4 weeks in genotypes 1 and 4) [43]. Resistance to cyclosporine and Debio 025 can be selected in vitro; however, mutations are scattered throughout NS5B and NS5A, and result in only a modest increase in $\mathrm{EC}_{50}$ [44]. No resistance mutations were documented in vitro during monotherapy or coadministration with IFN; thus, it appears the resistance barrier for cyclophilin inhibitors is high, similar to that described for NIs. Although these agents do not specifically target HCV viral proteins, they possess properties making them attractive as potential components of a STAT-C combination regimen.

\section{Will an All-STAT-C Regimen Work? Unique aspects of IFN and RBV}

The mechanism by which IFN-based therapy results in cure of chronic HCV is presumably by stimulating cellular antiviral mechanisms and immunomodulation. IFN therapy increases HCV-specific CD4 and CD8 T-cell responses, and these responses are associated with successful HCV therapy $[45,46]$. Although it seems clear that STAT-C agents can replace and even improve on the antiviral activity of IFN, whether the immunomodulatory effects are critical in achieving SVRs is unknown. HCV itself is immunosuppressive, both by direct interference with key cellular immune-response pathways (eg, NS3 protease cleavage of IFN- $\beta$ promoter stimulator-1) [47] and through a more nonspecific "immune exhaustion" associated with upregulation of the inhibitory molecule programmed death-1 [48]. Use of STAT-C agents should reverse these two forms of immunosuppression, but whether direct immune stimulation, as occurs with IFN, is needed for a durable cure remains the unanswered question while all-STAT-C regimens are pursued.

$\mathrm{RBV}$ also was a key component in preventing resistance and/or breakthrough during STAT-C combination therapy with IFN. Trials with STAT-C agents and PEG-IFN showed dramatic increases in viral breakthrough on therapy (PIs and NIs), significant increases in viral resistance to PIs, and increased relapse rates (PIs and NIs) when RBV is omitted or used at low doses $[10 \bullet \bullet, 12,32]$. RBV may act as both a mutagen and an immunomodulator and augments the second-phase of viral clearance, which is believed to correlate with infected hepatocyte loss [49]. Although it is now clear that RBV is necessary if a single STAT-C agent is combined with PEG-IFN, additional studies are needed to determine if an additional STAT-C agent can replace RBV.

\section{Future directions}

The much publicized INFORM-1 trial (ClinicalTrials. gov number NCT00801255) was the first to assess 
combination therapy with two STAT-C inhibitors in HCV-infected humans [50]. The NI R7128 (PSI-6130) was combined with the PI R7227 (ITMN-191) for 14 days. A decrease of about $5 \log$ in HCV RNA was seen in the three highest-dose groups. No viral rebound was seen during therapy, in contrast to PI monotherapy, where viral rebound can occur as early as day 3 . This suggests that the addition of a NI with a high barrier to resistance can prevent the emergence of PI-resistant $\mathrm{HCV}$ in the short term. One caveat to this assertion is that at this time, details on clonal analysis of viral populations for resistance mutations have not been presented. It also remains to be seen if subjects with breakthrough or relapse in the PEG-IFN/RBV rollover portion of the study will have PI or (less likely) NI resistance mutations in their viral quasispecies. Based on estimates by Perelson [51] from modeling of HCV replication and mutation rates in chronically infected humans, it seems unlikely that two STAT-C agents will be able to present the virus with a high enough resistance barrier to attain prolonged viral suppression and sustained responses.

Taking cues from the INFORM-1 study, what are some of the pressing issues as clinical trials of STAT-C combinations more forward? Developing a framework whereby novel combinations of STAT-C agents can be tested effectively in a timely manner is of utmost importance. Concepts learned during the testing and evolution of HIV-1 antiretroviral therapy must not be forgotten. National and international clinical trials networks, in close collaboration with the pharmaceutical industry and drug regulatory boards, will be necessary to push the field forward while maintaining patient safety. Because drug resistance is likely to be one of the major complications for an all-STAT-C regimen, the prospective, detailed evaluations of resistant viral variants are crucial. However, before theses studies can be accomplished, definitions and testing methodologies for the detection of resistant mutants need to be standardized. The HCV Drug Resistance Advisory Group was established to meet this goal.

\section{Conclusions}

Curing HCV infection with a STAT-C regimen presents formidable challenges because of viral characteristics that promote rapid resistance development. However, the large number of diverse candidate viral inhibitors and successful proof-of-concept clinical studies suggest that such a regimen is attainable. Unanswered questions surround the need for IFN and/or RBV as adjuncts to STAT-C agents, and the number of agents required to prevent resistance and viral breakthrough. Although nuances exist in the development and implementation of STAT-C combination therapy, many concepts fundamental to treating chronic viral infections such as HIV-1 undoubtedly apply.

\section{Disclosure}

No potential conflict of interest relevant to this article was reported.

\section{References and Recommended Reading}

Papers of particular interest, published recently,

have been highlighted as:

- Of importance

-• Of major importance

1. Fried MW, Shiffman ML, Reddy KR, et al.: Peginterferon alfa-2a plus ribavirin for chronic hepatitis $\mathrm{C}$ virus infection. N Engl J Med 2002, 347:975-982.

2. Falck-Ytter Y, Kale H, Mullen KD, et al.: Surprisingly small effect of antiviral treatment in patients with hepatitis $\mathrm{C}$. Ann Intern Med 2002, 136:288-292.

3. Muir AJ, Bornstein JD, Killenberg PG, Atlantic Coast Hepatitis Treatment Group: Peginterferon alfa- $\mathbf{2 b}$ and ribavirin for the treatment of chronic hepatitis $\mathrm{C}$ in blacks and non-Hispanic whites. N Engl J Med 2004, 350:2265-2271.

4. Torriani FJ, Rodriguez-Torres M, Rockstroh J, et al.: Peginterferon alfa-2a plus ribavirin for chronic hepatitis $\mathrm{C}$ virus infection in $\mathrm{HIV}$-infected patients. $\mathrm{N} \mathrm{Engl} \mathrm{J} \mathrm{Med}$ 2004, 351:438-450.

5. Simmonds P: Genetic diversity and evolution of hepatitis $\mathrm{C}$ virus-15 years on. J Gen Virol 2004, 85(Pt 11):3173-3188.

6. Soriano V, Perelson AS, Zoulim F: Why are there different dynamics in the selection of drug resistance in HIV and hepatitis B and C viruses? J Antimicrob Chemother 2008, 62:1-4. This article is a review of HCV virology, contrasted with HIV and $\mathrm{HBV}$, and how it is likely to impact the development of resistance to STAT-C inhibitors

7. Palella FJ Jr, Delaney KM, Moorman AC, et al.: Declining morbidity and mortality among patients with advanced human immunodeficiency virus infection. HIV Outpatient Study Investigators. N Engl J Med 1998, 338:853-860.

8. Tsantrizos YS: The design of a potent inhibitor of the hepatitis C virus NS3 protease: BILN 2061-from the NMR tube to the clinic. Biopolymers 2004, 76:309-323.

9. Lamarre D, Anderson PC, Bailey M, et al.: An NS3 protease inhibitor with antiviral effects in humans infected with hepatitis C virus. Nature 2003, 426(6963):186-189.

10.• Hézode C, Forestier N, Dusheiko G, et al.: Telaprevir and peginterferon with or without ribavirin for chronic HCV infection. N Engl J Med 2009, 360:1839-1850.

The authors present one of the first published studies on the SVR rates for HCV treated with a STAT-C protease inhibitor combined with PEG-IFN \pm RBV. The no-RBV group provides valuable information as STAT-C trials progress.

11. McHutchison JG, Everson GT, Gordon SC, et al.: Telaprevir with peginterferon and ribavirin for chronic HCV genotype 1 infection. N Engl J Med 2009, 360:1827-1838.

12. Kwo P, Lawitz E, McCone J, et al.: HCV SPRINT-1 final results: SVR 24 from a phase 2 study of boceprevir plus pegintron/ribavirin in treatment-naive subjects with genotype-1 chronic hepatitis C. J Hepatol 2009, 50(S1):S4.

13. Forestier N, Larrey D, Marcellin P, et al.: Antiviral activity and safety of ITMN-191 in combination with peginterferon alfa-2A and ribavirin in patients with chronic hepatitis $\mathrm{C}$ virus (HCV). J Hepatol 2009, 50(Suppl 1):S35.

14. Manns M, Gane E, Rodriguez-Torres M, et al.: MK-7009 significantly improves rapid viral response (RVR) in combination with pegylated interferon alfa- $2 \mathrm{~A}$ and ribavirin in patients with chronic hepatitis $\mathrm{C}(\mathrm{CHC})$ genotype 1 infection. J Hepatol 2009, 50(Suppl 1):S384.

15. Manns M, Reesink H, Moreno C, et al.: OPERA-1 trial: interim analysis of safety and antiviral activity of TMC435 in treatment-naive genotype $1 \mathrm{HCV}$ patients. J Hepatol 2009, 50(Suppl 1):S7. 
16. Jiang L, Gai Y, Middleton T, et al.: Potent HCV protease inhibitors with the potential for once-daily dosing and broad genotype coverage. J Hepatol 2009, 50(Suppl 1):S6.

17. Lin C, Lin $\mathrm{K}$, Luong $\mathrm{YP}$, et al.: In vitro resistance studies of hepatitis $\mathrm{C}$ virus serine protease inhibitors, VX-950 and BILN 2061: structural analysis indicates different resistance mechanisms. J Biol Chem 2004, 279:17508-17514.

18. Lin C, Gates CA, Rao BG, et al.: In vitro studies of crossresistance mutations against two hepatitis $\mathrm{C}$ virus serine protease inhibitors, VX-950 and BILN 2061. J Biol Chem 2005, 280:36784-36791.

19.• Sarrazin C, Kieffer TL, Bartels D, et al.: Dynamic hepatitis $\mathrm{C}$ virus genotypic and phenotypic changes in patients treated with the protease inhibitor telaprevir. Gastroenterology 2007, 132:1767-1777.

This article provides the first detailed clonal analysis of HCV proteaseresistant variants emerging during protease-inhibitor monotherapy.

20. He Y, King MS, Kempf DJ, et al.: Relative replication capacity and selective advantage profiles of protease inhibitor-resistant hepatitis C virus (HCV) NS3 protease mutants in the HCV genotype $1 \mathrm{~b}$ replicon system. Antimicrob Agents Chemother 2008, 52:1101-1110.

21. Kuntzen T, Timm J, Berical A, et al.: Naturally occurring dominant resistance mutations to hepatitis $\mathrm{C}$ virus protease and polymerase inhibitors in treatment-naïve patients. Hepatology 2008, 48:1769-1778.

22. Bartels DJ, Zhou Y, Zhang EZ, et al.: Natural prevalence of hepatitis C virus variants with decreased sensitivity to NS3.4A protease inhibitors in treatment-naive subjects. J Infect Dis 2008, 198:800-807.

This article is a detailed analysis of preexisting protease inhibitor resistance muations. The authors suggest that preexisting R155K mutants may be particularly problematic for treatment with current HCV protease inhibitors.

23. Susser S, Forestier N, Welker M, et al.: Detection of resistant variants in the hepatitis C virus NS3 protease gene by clonal sequencing at long-term follow-up in patients treated with boceprevir. J Hepatol 2009, 50(Suppl 1):S7-S7.

24. Bressanelli S, Tomei L, Roussel A, et al.: Crystal structure of the RNA-dependent RNA polymerase of hepatitis C virus. Proc Natl Acad Sci U S A 1999, 96:13034-13039.

25. Watashi K, Shimotohno K: Chemical genetics approach to hepatitis $\mathrm{C}$ virus replication: cyclophilin as a target for anti-hepatitis C virus strategy. Rev Med Virol 2007, 17:245-252.

26. Pauwels F, Mostmans W, Quirynen LM, et al.: Binding-site identification and genotypic profiling of hepatitis $\mathrm{C}$ virus polymerase inhibitors. J Virol 2007, 81:6909-6919.

27. Carroll SS, Tomassini JE, Bosserman M, et al.: Inhibition of hepatitis C virus RNA replication by 2 '-modified nucleoside analogs. J Biol Chem 2003, 278:11979-11984.

28. McCown MF, Rajyaguru S, Le Pogam S, et al.: The hepatitis $\mathrm{C}$ virus replicon presents a higher barrier to resistance to nucleoside analogs than to nonnucleoside polymerase or protease inhibitors. Antimicrob Agents Chemother 2008, 52:1604-1612.

29. Di Marco S, Volpari C, Tomei L, et al.: Interdomain communication in hepatitis $\mathrm{C}$ virus polymerase abolished by small molecule inhibitors bound to a novel allosteric site. J Biol Chem 2005, 280:29765-29770.

30. Wang M, Ng KKS, Cherney MM, et al.: Non-nucleoside analogue inhibitors bind to an allosteric site on HCV NS5B polymerase. Crystal structures and mechanism of inhibition. J Biol Chem 2003, 278:9489-9495.

31. Lawitz E, Nguyen T, Younes Z, et al.: Clearance of HCVRNA with valopicitabine (NM) plus peg-interferon in treatment-naive patients with $\mathrm{HCV}-1$ infection: results at 24 and 48 weeks. J Hepatol 2007, 46(Suppl 1):S9-S9.
32. Pockros P, Nelson D, Godofsky E, et al.: High relapse rate seen at week 72 for patients treated with R1626 combination therapy. Hepatology 2008, 48:1349-1350.

33. Klumpp K, Kalayanov G, Ma H, et al.: 2'-deoxy-4'-azido nucleoside analogs are highly potent inhibitors of hepatitis $\mathrm{C}$ virus replication despite the lack of 2'-alpha-hydroxyl groups. J Biol Chem 2008, 283:2167-2175.

34. Lalezari J, Gane E, Rodriguez-Torres M, et al.: Potent antiviral activity of the HCV nucleoside polymerase inhibitor R7128 with peg-IFN and ribavirin: interim results of R7128 $500 \mathrm{mg}$ bid for 28 days. J Hepatol 2008, 48(Suppl 2):S29-S29.

35. Lawitz E, Rodriguez-Torres M, DeMicco M, et al.: Antiviral activity of ANA598, a potent non-nucleoside polymerase inhibitor, in chronic hepatitis C patients. J Hepatol 2009, 50(Suppl 1):S384-S384.

36. Lawitz E, Cooper C, Rodriguez-Torres M, et al.: Safety, tolerability and antiviral activity of $\mathrm{VCH}-916$, a novel non-nucleoside HCV polymerase inhibitor in patients with chronic HCV genotype-1 infection. J Hepatol 2009, 50(Suppl 1):S37-S37.

37. Le Pogam S, Seshaadri A, Kosaka A, et al.: Existence of hepatitis C virus NS5B variants naturally resistant to nonnucleoside, but not to nucleoside, polymerase inhibitors among untreated patients. J Antimicrob Chemother 2008, 61:1205-1216.

This article provides a detailed analysis of preexisting NS5B polymerase-resistant mutants. The work suggests that nucleoside inhibitor mutants do not preexist in significant amounts. Preexisting nonnucleoside inhibitor resistance may be a significant hurdle to their widespread implementation.

38. Tomei L, Altamura S, Paonessa G, et al.: HCV antiviral resistance: the impact of in vitro studies on the development of antiviral agents targeting the viral NS5B polymerase. Antivir Chem Chemother 2005, 16:225-245.

39. Le Pogam S, Kang H, Harris SF, et al.: Selection and characterization of replicon variants dually resistant to thumb- and palm-binding nonnucleoside polymerase inhibitors of the hepatitis C virus. J Virol 2006, 80:6146-6154.

40. Coelmont L, Paeshuyse J, Windisch MP, et al.: Ribavirin antagonizes the in vitro anti-hepatitis $C$ virus activity of 2'-C-methylcytidine, the active component of valopicitabine. Antimicrob Agents Chemother 2006, 50:3444-3446.

41. Darling JM, Fried MW: Nitazoxanide: beyond parasites toward a novel agent for hepatitis C. Gastroenterology 2009, 136:760-763.

42. Bacon B, Shiffman M, Lim J, et al.: Phase 2 randomized, double-blind, placebo-controlled study of nitazoxanide plus peginterferon and ribavirin in HCV genotype 1 patients: interim analysis shows increase in EVR. J Hepatol 2009, 50(Suppl 1):S381-S381.

43. Flisiak R, Feinman SV, Jablkowski M, et al.: The cyclophilin inhibitor Debio 025 combined with PEG IFN-alpha2a significantly reduces viral load in treatment-naive hepatitis C patients. Hepatology 2009, 49:1460-1468.

44. Fernandes F, Poole DS, Hoover S, et al.: Sensitivity of hepatitis $\mathrm{C}$ virus to cyclosporine $\mathrm{A}$ depends on nonstructural proteins NS5A and NS5B. Hepatology 2007, 46:1026-1033.

45. Kamal SM, Fehr J, Roesler B, et al.: Peginterferon alone or with ribavirin enhances HCV-specific CD4 T-helper 1 responses in patients with chronic hepatitis C. Gastroenterology 2002, 123:1070-1083.

46. Löhr HF, Schmitz D, Arenz M, et al.: The viral clearance in interferon-treated chronic hepatitis $\mathrm{C}$ is associated with increased cytotoxic T cell frequencies. J Hepatol 1999, 31:407-415. 
47. Johnson CL, Owen DM, Gale M: Functional and therapeutic analysis of hepatitis $\mathrm{C}$ virus NS3.4A protease control of antiviral immune defense. J Biol Chem 2007, 282:10792-10803.

48. Golden-Mason L, Palmer B, Klarquist J, et al.: Upregulation of PD-1 expression on circulating and intrahepatic hepatitis $\mathrm{C}$ virus-specific CD8+ T cells associated with reversible immune dysfunction. J Virol 2007, 81:9249-9258.

49. Dahari H, Ribeiro RM, Perelson AS: Triphasic decline of hepatitis C virus RNA during antiviral therapy. Hepatology 2007, 46:16-21.
50. Gane E, Roberts S, Stedman C, et al.: First-in-man demonstration of potent antiviral activity with a nucleoside polymerase (R7128) and protease (R7227/ITMN-191) inhibitor combination in HCV: safety, pharmacokinetics, and virologic results from INFORM-1. J Hepatol 2009, 50(Suppl 1):S380-S380.

51. Perelson A: New insights into the viral dynamics of HCV infection: can virologic cure be achieved without interferon and ribavirin. Presented at the 16th Conference on Retroviruses and Opportunistic Infections. Montreal, Canada: February 8-11, 2009. Available at http://www. retroconference.org/2009/Abstracts/36557.htm. Accessed September 2009. 